\title{
Web Based Home Automation System
}

\author{
Nayera Nahvi \\ Department of E\&C Engineering, Islamic University of science \& Technology Awantipora, \\ J\&K India \\ E-mail address: nahvinayera@gmail.com
}

\begin{abstract}
Web Based Home Automation System provides the robust solution for communication to meet the increasing demand of the people. The idea was to design and develop such a system that will convert a manually controlled home system to a smart home system that will be controlled from any remote location by using web site (using internet). This system enables a user to get the status of all the devices in home (that are connected to this system) and it will also provide a great control over the devices even if the user is at a remote location. It increases the efficiency and decreases the response time to user.
\end{abstract}

Keywords:Web BasedHome automation system (WBHAS), Smart Home Hub(SHB), Active Server Pages(ASP), domotics, personal computer (PC).

\section{INTRODUCTION}

Home automation or domotics is the automation of the home to make it a smart home.Due to significant advances in Internet and computer technology, the Internet has started to serve as a medium that be used in home automation, which provides many features ranging from efficient use ofenergy to increased comfort [1].Due to advent of advanced computer and wideband network, the personal computer based environment seems to be very suitable platform for system integration. The PC's can be linked by the network and are capable of powerful computation and easy display [2].Fei-Yue WANG et al. proposed an Internetapplication that allows local and remote monitoringand control of a home [3]. The applicationwas built on a single chip system with networkinterface but due to the hardware limitation of the singlechip system, the system is less flexible and scalable, although the system embedded TCP/IPprotocol and could be accessed and controlled over theInternet.

We have developed a system that receives control commands through internet and then after processing the received data, it transfers that data to automation unit. To provide versatility, the controlling source will be supported, through which we can transfer control command to the automation unit. The source is a web application. The home web server is a PC, connected to theinternet. The web provides a remote access to the automation unit. A modem is connected to our system to receive the commands from the user. The user is authenticated before processing any of the action. System analysis was done using prototyping and object oriented methodology. The technology that has been used is MS Visual Studio.NET 2005 and MS SQL server 2005. 


\section{WBHAS}

We have designed a method that enables the user to get access to home devices if he is at a remote location in an efficient way in order to reduce the improper utilization of valuable resources like human effort, time and cost. It consists of two modules i) Smart Home Hub (SHB) ii) Automation unit.

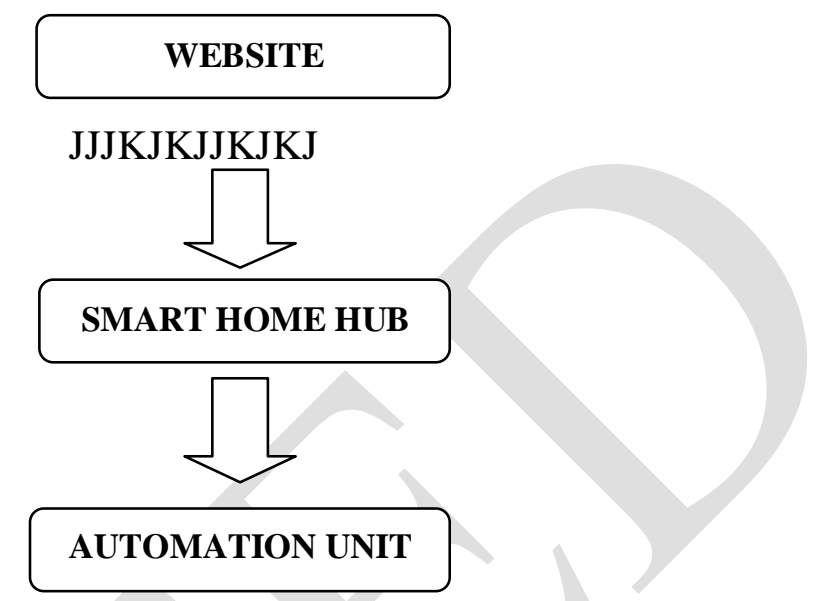

Fig 1.Block diagram of WBHAS

\section{A. Smart Home Hub}

Smart Home Hub is the central part of our system that is responsible for receiving updates from website. After receiving all those messages successfully SHB transmits command to the automation unit by using parallel interface. It is a web application which is capable of:

\section{i) Changing current status of devices}

To change the current status of devices a web interface is provided to the user. User can change the status of thedevices using these applications and SHB will send a message of change for the current status of devices to the automation unit in order to make the necessary changes.

\section{ii) Maintaining accounts}

The accounts are maintained by SHB by keeping the information about authentic users, so as to keep the record of the users for the security of the system.

\section{iii) Interacting with the automation unit}

SHB generates a series of code patterns which are passed to the automation unit through parallel communication. Then these code patterns are implemented by changing the status of the devices by performing switching of the devices.

\section{B. Automation Unit}

This is a hardware unit. Automation unit has a parallel interface for the purpose of communicating with the smart home hub. It receives data from the smart home hub that contains the information about the status of the devices attached to the automation unit. All the devices are connected to automation unit and switching of these devices is done after 
receiving message from smart home hub. At maximum we can attach 31 devices to our system. It is responsible for validating the messages received from SHB.

\section{SOFTWARE DESIGN}

It has been implemented using Microsoft SQL server 2005 and Active server pages (ASP) for web programming. ASP is used to provide a user interface and will be displayed using web browser while SQL is used for data base. We have developed the home automation website pages that allow the users to interactively access the home automation system remotely.

\section{A. Home Automation Website}

The server works on Windows 2000 professional OS.The requests of the remote users are submitted by using a standard web browser. The user can send the command to the system to get the current status of the device in the home, whether it is ON/OFF. User can also change the status of the device using the website. The data base design of our system is shown below in Fig 2.

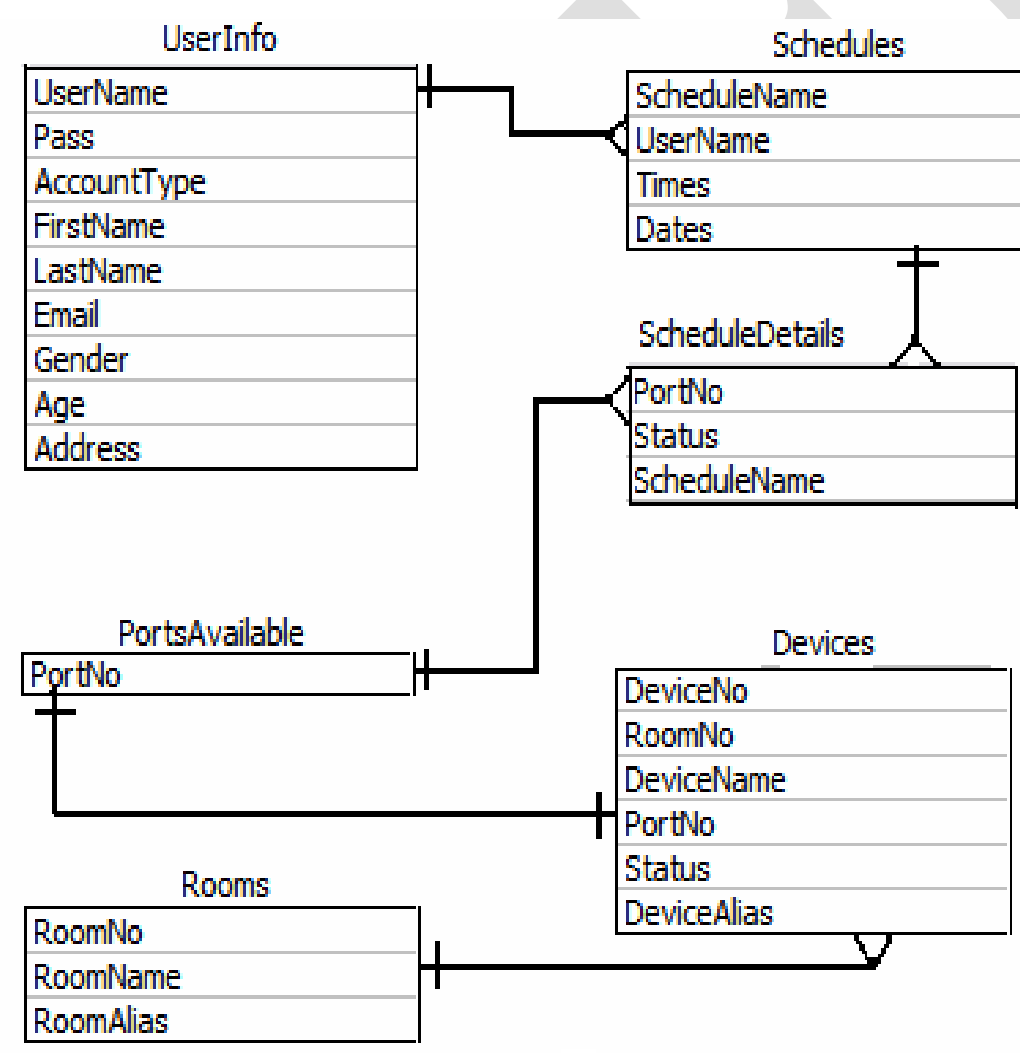

Fig 2. Data base design model

During the requirement analysis phase the following use case diagrams were depicted to represent the system. Fig 3 andfig 4 below show the various use case diagrams. 


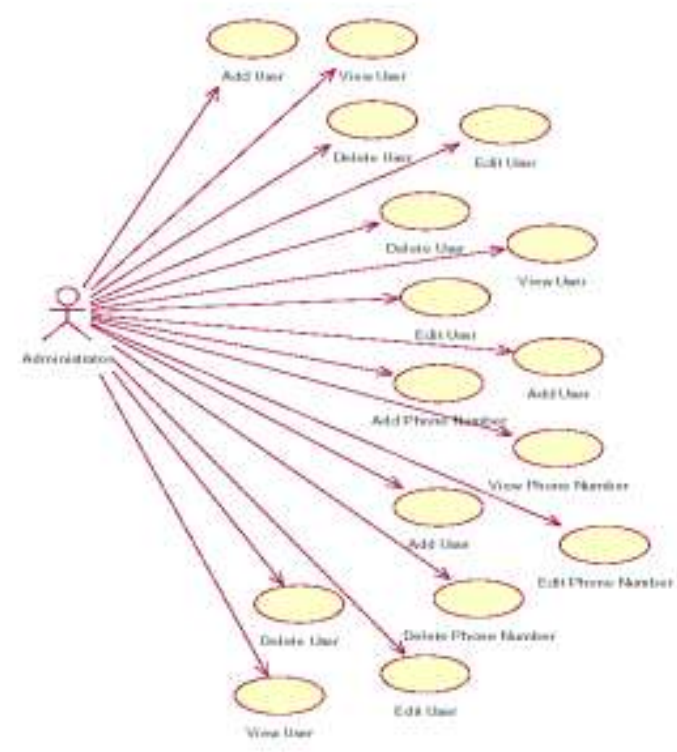

Fig 3. Use case diagram

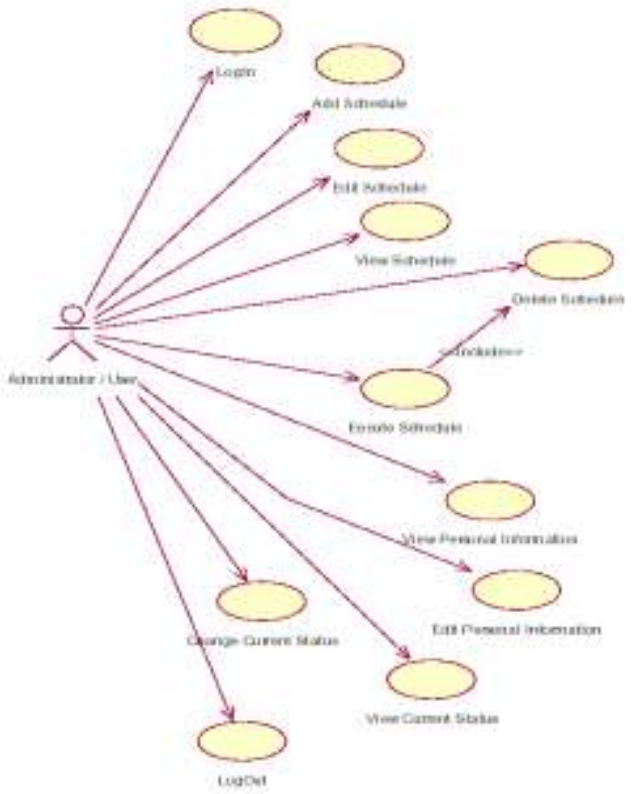

Fig 4. Use case diagram

If the system fails to receive the valid status of the login name and password the person is prompted to re- enter login name and password. Fig 5 and fig 6.,show the snapshots of home page and login page.

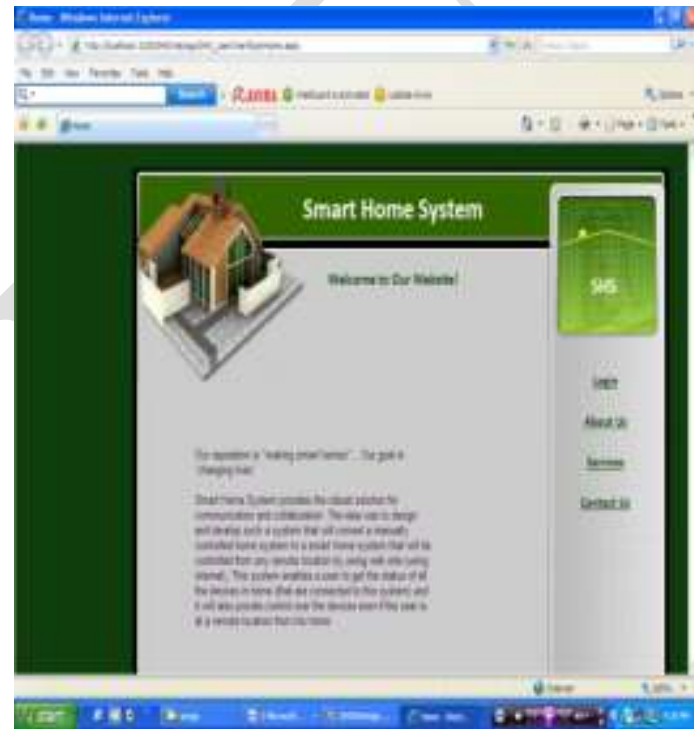

Fig 5. Home page

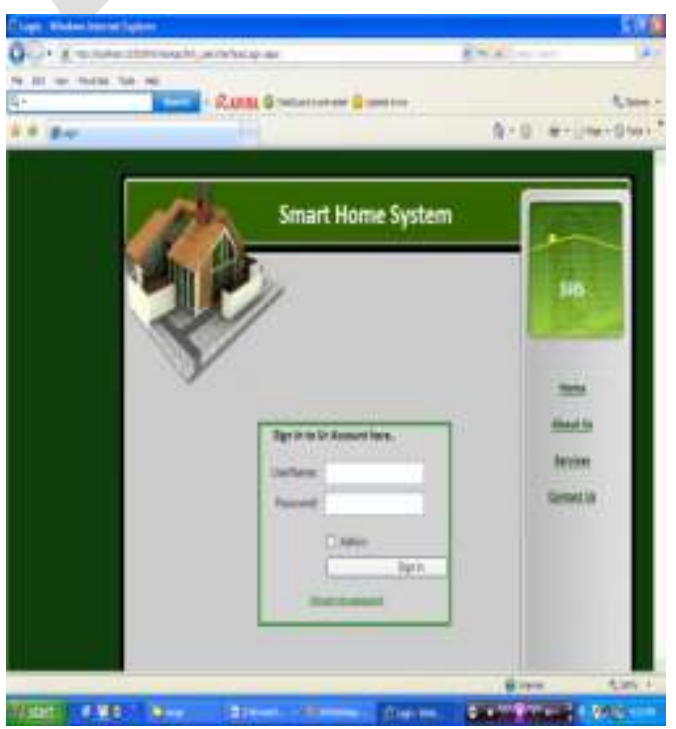

Fig 6. Login page

Fig 7 and fig 8.show the snapshots of the list of services provided by WBHAS and device status respectively. 


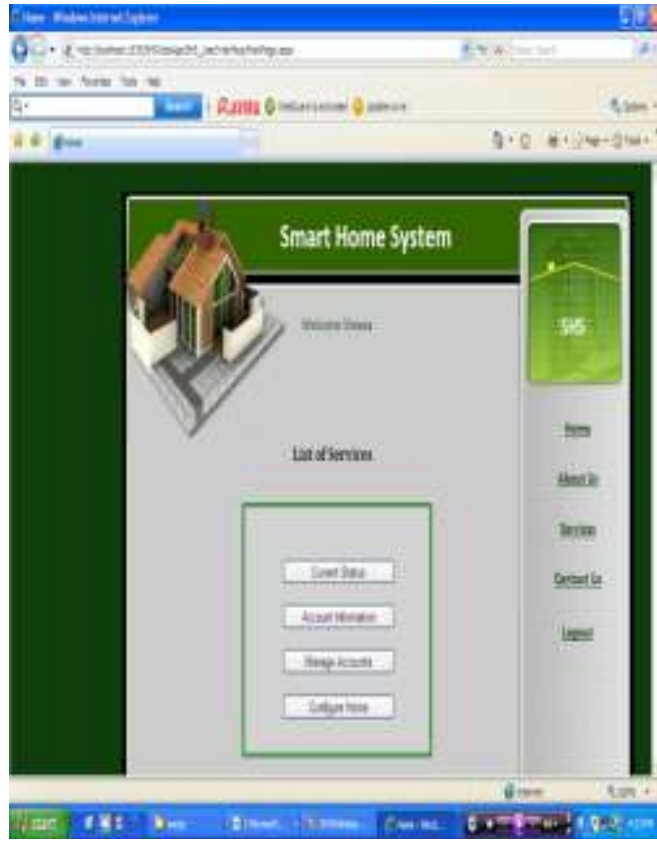

Fig 7. List of services

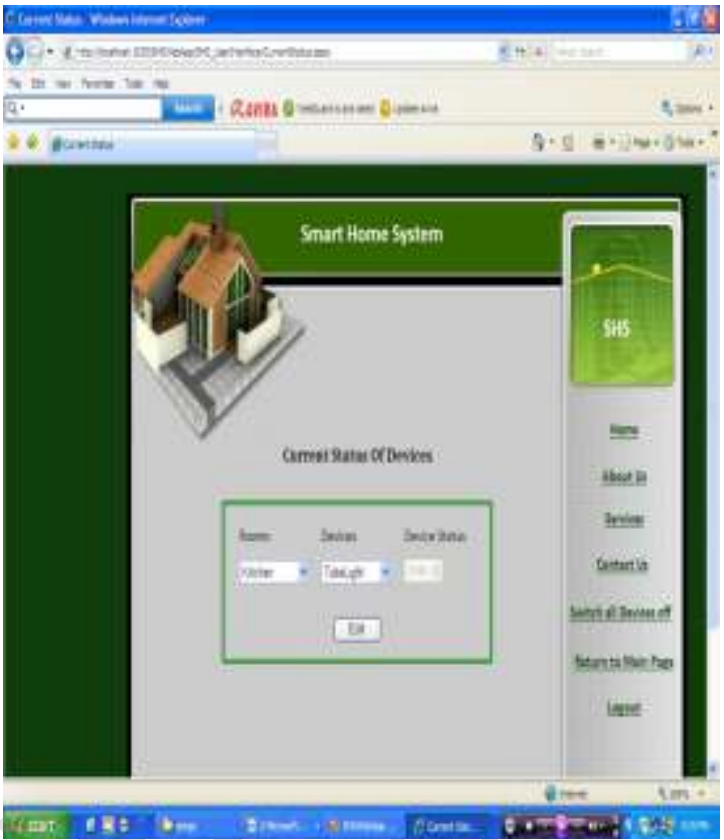

Fig 8. Current status of device

\section{HARDWARE ARCHITECTURE}

HAS consists of two hardware components, the 8051 microcontroller and the home web server. The home web server is a PC, connected to the internet. A modem is connected to the PC to receive the commands from the user. The PC will send the serially through its serial port, the voltage levels of which areconverted to make them compatible with the microcontroller. MAX232 will then send the converted levels tothe programmed microcontroller which in turn will send them to its output pins to change the status of the connecteddevices via external circuitry i.e., resistors, LED's and relays.

\section{CONCLUSION}

We have proposed a method which shows that it is possible to control home devices via the Internet and with no additional wiring. It will operate 3 important base case devices, a tube light, a bulb, and heater. These devices will be controlled through a server running the web page to and equipped with a device that transmits the signals to the appliances and the program to control the devices.This will assist in energy safety and security. The developed automated home can be accessed and modified from any place that has access to the internet service provider and is cost efficient and less time consuming.

\section{REFERENCES}

[1] K.Tan, T. Lee ,C. Yee Soh, "Internet-Based Monitoring of Distributed Control SystemsAn Undergraduate Experiment", IEEE Transactions on Education, Vol. 45, No. 2, May 2002.

[2] N.S.Liang, L.C.Fu, C.L.Wu“"An Integrated, Flexible and Internet-based Control Architecture for Home Automation System",The Internet Era Proceeding of IEEE International Conference on Robotics and Automation, Vol.4, May 2002, pp $1101-1106$. 
[3] F.Y. Wang, Y. Lin, Q. Wu, P.M Fu,C.Yeo, "Architecture and implementation of intelligent control systems for smart consumer appliances via Internet", Proceedings of the IEEE International Conference on Systems, Man and Cybernetics, Vol 5, Oct2000,pp 599-602.

[4] Andrew S. Tanenbaum, Computer Networks, Prentice Hall of India.

[5] The 8051 microcontroller by Kenneth J. Ayala.

[6] www.keil.com/forum/docs 